\title{
Lidar Data Analysis for Time to Headway Determination in the DriveSafe Project Field Tests
}

\author{
İlker Altay, ${ }^{1}$ Bilin Aksun Güvenç, ${ }^{2}$ and Levent Güvenç ${ }^{2}$ \\ ${ }^{1}$ Department of Mechanical Engineering, Istanbul Technical University, Istanbul TR-34437, Turkey \\ ${ }^{2}$ Faculty of Engineering and Architecture, Okan University, Istanbul TR-34959, Turkey \\ Correspondence should be addressed to Levent Güvenç; levent.guvenc@okan.edu.tr
}

Received 9 October 2012; Revised 21 December 2012; Accepted 30 December 2012

Academic Editor: Tang-Hsien Chang

Copyright ( 2013 Ilker Altay et al. This is an open access article distributed under the Creative Commons Attribution License, which permits unrestricted use, distribution, and reproduction in any medium, provided the original work is properly cited.

\begin{abstract}
The DriveSafe project was carried out by a consortium of university research centers and automotive OEMs in Turkey to reduce accidents caused by driver behavior. A huge amount of driving data was collected from 108 drivers who drove the instrumented DriveSafe vehicle in the same route of $25 \mathrm{~km}$ of urban and highway traffic in Istanbul. One of the sensors used in the DriveSafe vehicle was a forward-looking LIDAR. The data from the LIDAR is used here to determine and record the headway time characteristics of different drivers. This paper concentrates on the analysis of LIDAR data from the DriveSafe vehicle. A simple algorithm that only looks at the forward direction along a straight line is used first. Headway times based on this simple approach are presented for an example driver. A more accurate detection and tracking algorithm taken from the literature are presented later in the paper. Grid-based and point distance-based methods are presented first. Then, a detection and tracking algorithm based on the Kalman filter is presented. The results are demonstrated using experimental data.
\end{abstract}

\section{Introduction}

(National Highway Traffic Safety Administration) NTHSA reported six million vehicle crashes in 2005 that resulted in 43,000 dead and 2.5 million injured people in the USA [1]. Moreover, (International Road Traffic and Accident Database) IRTAD reported 40,000 dead and 1.4 million injured people all over Europe in the same year. Driver mistakes caused 90 percent of these crashes [2]. Driving dynamics should be investigated to prevent accidents caused by driver mistakes before they take place. Driving dynamics is the interaction between vehicle, road conditions, and the driver [3]. The theoretical model of driving behaviour based on a microsimulator is presented in [4] where the driver error is separated into perception, decision-making, and action parts. According to [5], the development in passive and active safety systems, car accidents, and fatalities per distance decrease as the total distance travelled per year increases.

The DriveSafe project was started to reduce accidents caused by driver behavior with the collaboration of OTAM, Sabanci University, İstanbul Technical University, Ford A.Ş., Renault A.Ş., and Tofas A.Ş. A huge amount of driving data was collected in 2006. A total of 108 drivers, 89 men and 19 women, drove the DriveSafe vehicle in the same route of $25 \mathrm{~km}$ in İstanbul $[6,7]$. This instrumented vehicle, used for multimodal data collection, is a Renault Megane see (Figure 1) equipped with a large array of sensors and a data acquisition system. It includes two cameras looking at the driver, one camera looking at the road and several microphones. Additional sensors include an EEG and a heart beat sensor that are used for validation of driver state, GPS and a laser scanner. Also, gas, brake pedal pressure, steering angle, and vehicle speed data were collected through the vehicle CAN bus $[6,7]$.

Time to headway is an important driving characteristic that shows significant changes between drivers if other conditions like the road (urban, highway), traffic (dense, light), and so forth are kept the same. Time to headway (TTH) is the main controlled variable in Advanced Driver Assistance Systems (ADAS) like Adaptive Cruise Control (ACC) and Cooperative Adaptive Cruise Control (CACC). In ACC and CACC, the claim is that safer TTH values are used as compared to manual speed control by the driver [8]. It is, 


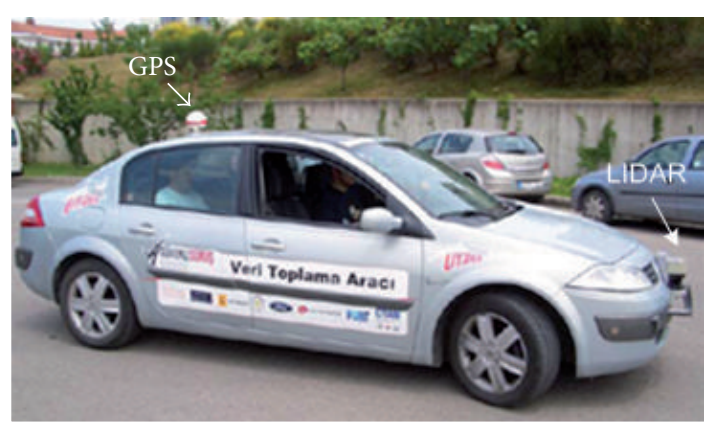

FIGURE 1: DriveSafe project vehicle named "UYANIK."

therefore, important to first analyze TTH characteristics in manual driving. This will form a benchmark that TTH values regulated with ACC and CACC systems can be compared against.

This paper concentrates on extracting TTH values from the data collected in the DriveSafe project field tests. The Lidar in front of the vehicle (see Figure 1) is chosen as the sensor that will be used for TTH determination. This paper presents both a simple approach and detection and trackingbased approach for computing TTH values based on front looking Lidar data.

TTH has been analyzed in detail in previous work in the literature. In most of these references, TTH data is computed either using a simulator or measures based on sensors embedded in the road and correlated with traffic congestion $[9,10]$. In other references, TTH is investigated as a part of an ACC or CACC system, and TTH values for manual driving are not treated $[8,11]$. In contrast to the abovementioned and similar references, the TTH computations in this paper are for manual driving by a human driver. TTH is used to characterize the driving characteristics of drivers and is not used to investigate traffic congestion. Instead of using sensors in the road or a radar (for vehicles with ACC), a Lidar is used in this paper to determine TTH values.

The outline of the rest of the paper is as follows. The DriveSafe experiments and a simple approach to TTH computation are presented in Section 2. The grid-based method and the point distance-based segmentation method are outlined in Section 3. The Kalman filter approach to detection and tracking of vehicles in front is outlined in Section 4. The Kalman filter-tracking results are presented in Sections 5 and 6. The paper ends with conclusions.

\section{Driver Characteristics Based on Collected Lidar Data}

A single-layer laser scanner made by SICK with a $1 \mathrm{~Hz}$ sampling frequency and a range of view of 81 meters was used to detect the distance to the vehicle in front of the DriveSafe data collection vehicle. This distance and the recorded speed of the experimental vehicle can be used to determine the headway time. Figure 2 shows two different plots showing one Lidar scan of the ego vehicle's front side. This section presents analysis of the time to headway (TTH) characteristics of

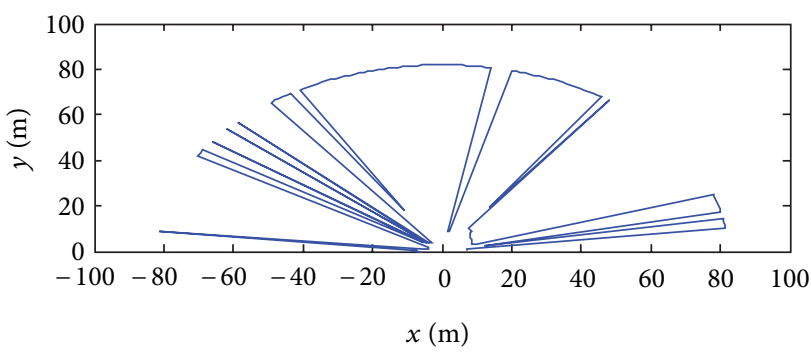

(a)

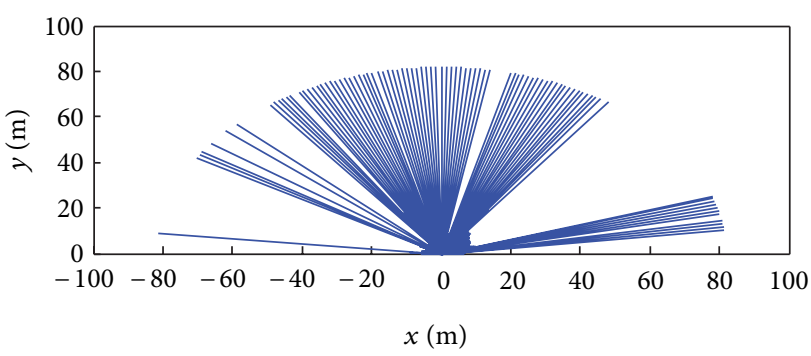

(b)

FIGURE 2: Plotting samples of lidar data.

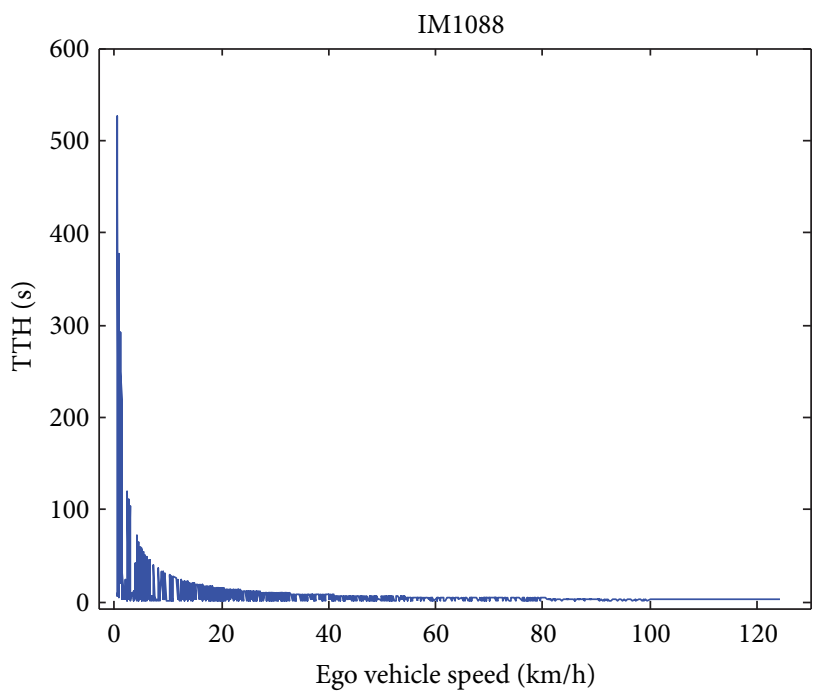

FIGURE 3: TTH versus ego vehicle speed.

different drivers in the DriveSafe experiments. TTH is the amount of time the experimental vehicle would take at its current speed and vehicle-to-vehicle distance to reach the vehicle in front assuming that the vehicle in front is stationary. Using a simplistic approach, the Lidar data at 90 degrees (vertical line from $x=0 \mathrm{~m}$ ), that is, directly ahead of the ego vehicle, is selected for relative distance calculation in Figure 2.

Time to headway is the ratio of relative distance to ego vehicle speed. In the literature, there is a 2 -second rule for a safe headway in manual driving. The driving situation is safe if TTH is equal to or more than 2 seconds. It is possible to use lower headway times on the order of 0.6 to $2 \mathrm{sec}$ in 


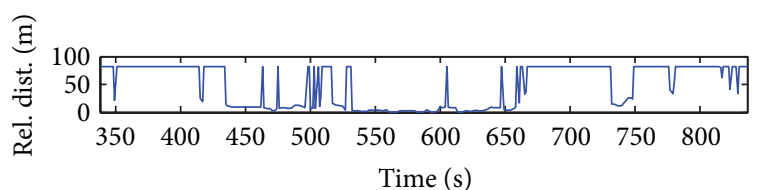

(a)

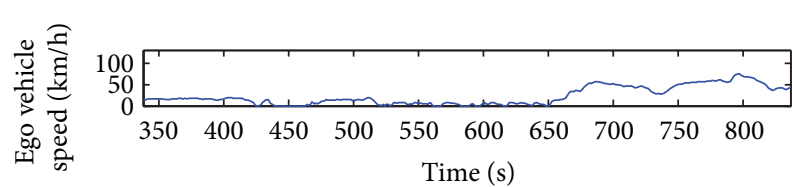

(c)

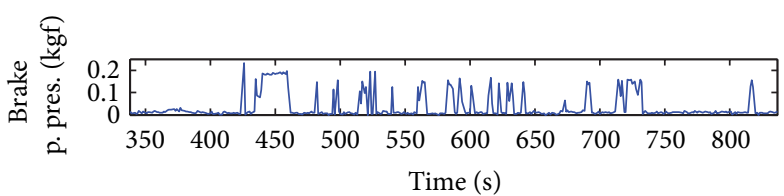

(b)

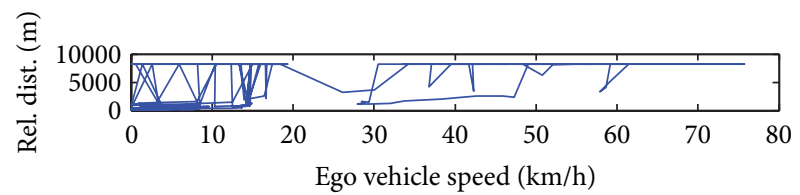

(d)

FIGURE 4: CAN bus and lidar data plots to investigate driver behavior.
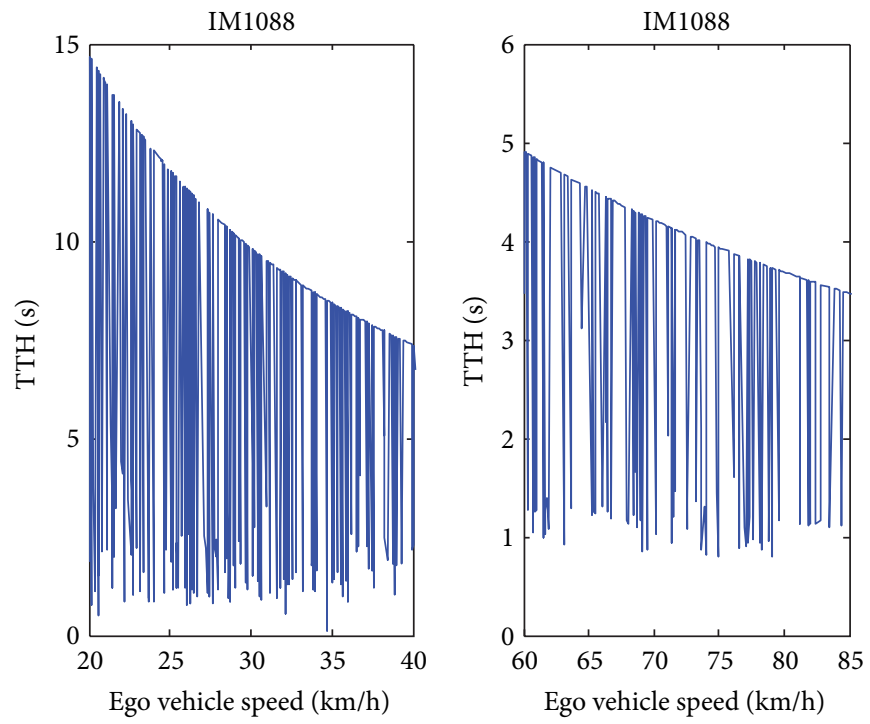

FIgURE 5: TTH versus different ego speed vehicle ranges.

TABLE 1: Drivers statistical information for TTH (in seconds).

\begin{tabular}{lcccc}
\hline & Min & Max & Mean & Std Dev. \\
\hline IM1084 & 0.379 & 8.210 & 1.247 & 0.849 \\
IM1085 & 0.298 & 6.424 & 1.271 & 0.825 \\
IM1088 & 0.514 & 4.988 & 1.561 & 0.786 \\
IM1089 & 0.736 & 4.197 & 2.031 & 0.576 \\
\hline
\end{tabular}

semiautomated driving using Cooperative Adaptive Cruise Control (CACC) or Adaptive Cruise Control (ACC), for example.

2.1. Time to Headway (TTH). In Figure 3, how TTH changes with the ego vehicle speed are shown for the driver with data code IM1088. M denotes a male driver and 1088 denotes the 88th driver in the dataset. The data in Figure 2 shows that the upper value of TTH decreases as the ego vehicle increases speed. If one concentrates on the lower envelope of the data in Figure 3, however, it is seen that an almost constant lower bound on TTH with a slightly increasing trend with increasing speed is used by this driver.

Typical data used in the computations is displayed in Figure 4. This data includes relative distance (Lidar), brake pressure $(\mathrm{CAN})$, and ego vehicle speed (CAN) versus time. The last subplot in Figure 4 shows the relative distance between the vehicles versus ego vehicle speed. Taking a look at the lower envelope of the data in that subplot of the figure shows that relative distance kept by the driver increases with vehicle speed, as expected.

If Figure 3 is zoomed and different speed ranges are concentrated upon as in Figure 5, it can be seen that the driver is using a lower bound of $1 \mathrm{sec}$ at higher speeds, which can be considered to be dangerous for manual driving. Note that the higher speeds in the zoomed plot in Figure 5 correspond to highway driving. These results illustrate the usefulness of equipping vehicles with ACC and CACC for safer vehicle following and with Collision Warning (CW) and Collision Avoidance (CA) as accident preventing measures in the case of manual driving. 


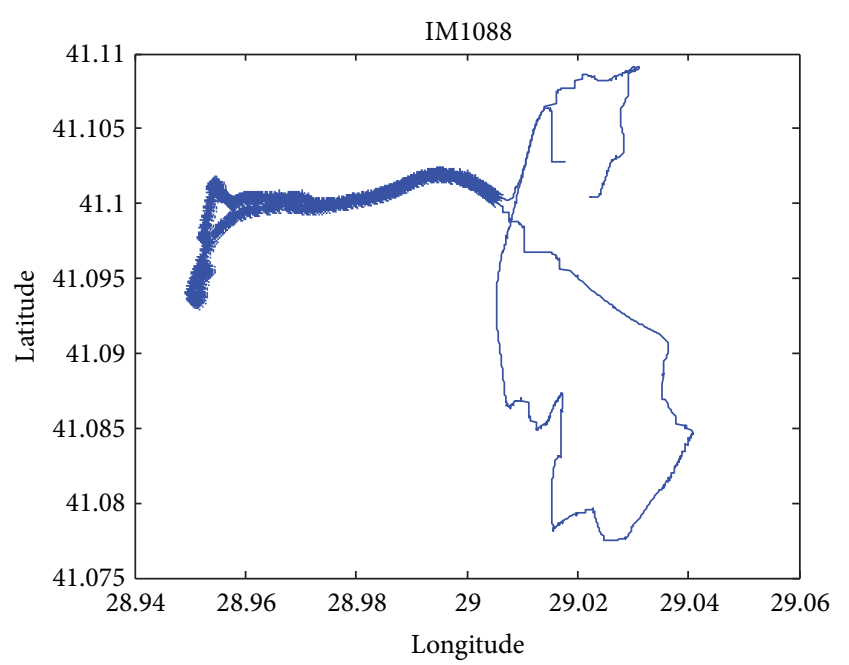

FIGURE 6: Bold points represent D100 highway.

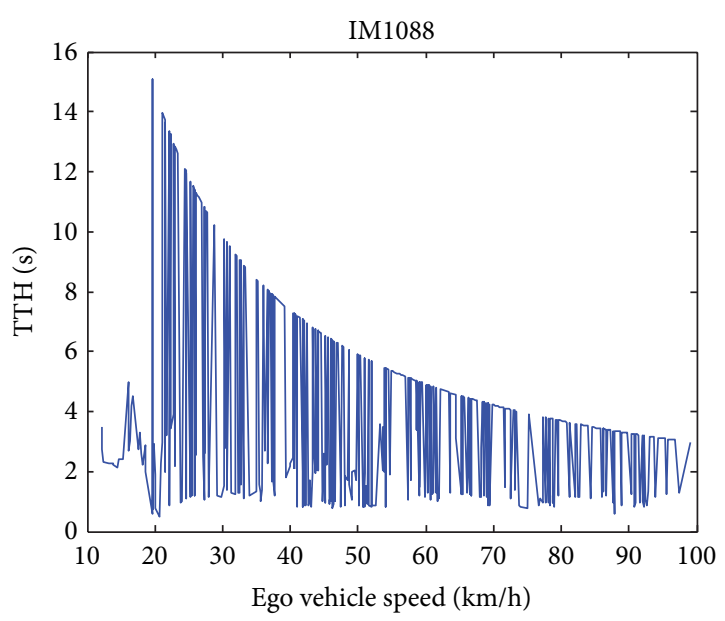

FigURE 7: TTH versus ego vehicle speed in D100 highway.

GPS data was also collected with the DriveSafe vehicle. This GPS data when placed on a map allowed us to extract highway driving part of the data. The GPS location data for the same driver is illustrated in Figure 6 where the highway driving part of the trajectory is marked separately.

Figure 7 shows the computed TTH values versus ego vehicle speed in the highway driving part of the data. There is no information below $10 \mathrm{~km} / \mathrm{h}$ of ego vehicle speed as traffic congestion did not occur during highway driving. Minimum TTH is detected as $0.5143 \mathrm{sec}$. This, of course, is a very low value and is not safe in manual driving. The average TTH values are much higher. Average TTH values of about $2 \mathrm{sec}$, which correspond to safe manual driving, are observed at high speeds $(70-100 \mathrm{~km} / \mathrm{hr})$ in Figure 7 . The maximum TTH values used are pretty high and range from $3.5 \mathrm{sec}$ at $100 \mathrm{~km} / \mathrm{hr}$ to $14 \mathrm{sec}$ at $20 \mathrm{~km} / \mathrm{hr}$. These high variations in TTH values imply that the use of semiautomated ACC or CACC driving in platoons of vehicles would help driving at both the individual vehicle and traffic flow levels. This is based on the expectation that ACC and CACC equipped vehicles will be able to reach TTH values of 1.0 to $0.6 \mathrm{sec}$ with very little scatter in TTH at individual speeds as compared to the very large scatter in the vertical direction in the manual driving example of Figure 7.

Minimum following distance differs for each driver. How relative distance between vehicles changes with ego vehicle speed is displayed in Figure 8. It is seen that the lower bound of the relative distance increases with speed.

\section{Lidar Data Processing}

The previous section concentrated on using the Lidar for vehicle-to-vehicle distance-and TTH calculations where only the 90 degrees (directly across the ego vehicle) information from the Lidar was used. In contrast, the data in the complete 180 degrees sweep of the Lidar is used in this section to detect vehicles in front and to detect road limits on both sides due to vehicles on other lanes. The grid-based method and point distance-based method for vehicle segmentation in lidar data processing that are both available in the literature are used and presented in this section.

3.1. Grid-Based Method. The laser scans the area in front of the ego vehicle. This area is divided into grids. In this study, the grid resolution is $1 \mathrm{~m}^{2}$. It is necessary to improve this resolution to be able to distinguish smaller objects. If a scan falls within a grid, then the probability of having an object inside it is assigned the value of 0.9. This is called grid occupancy probability. The cells before an occupied cell are assigned an occupancy probability of 0.1 . The cells that have unknown state are assigned an occupancy probability of 0.5. The grid-based method is generally used in image processing.

Figure 9 displays the grid-based representation of a Lidar scan. White cells at number 83 in the $x$ axis show the ego vehicle. After the cells are assigned occupancy probabilities, the connected components algorithm (CCA) in [12] is used to determine whether cells are connected or not. This method gives object size and information on whether the detected object is a vehicle, truck, or road limit. In Figure 9, the different colored grid points possibly represent objects (sides of the road and vehicles in front). Note that the grid-based method gives a coarse image of the vehicles in front and should not be preferred for TTH computations.

3.2. Point-Distance-Based Segmentation Method. Segments are a set of planar range measurements (points) close to each other, with the property that they have a high probability of belonging to one single object. There exist many segmentation methods in the literature. The point-distance-based segmentation method (PDBS) as presented in [13] is selected and used here. It is based on calculating the distance between two consecutive scanned points which is then compared to a threshold value.

The $Y$ axis represents the front of the ego vehicle in Figure 10. If the distance between two successive scan points given by

$$
D\left(r_{i}, r_{i+1}\right)=\sqrt{r_{i}^{2}+r_{i+1}^{2}-2 r_{i} r_{i+1} \cos \Delta \alpha}
$$



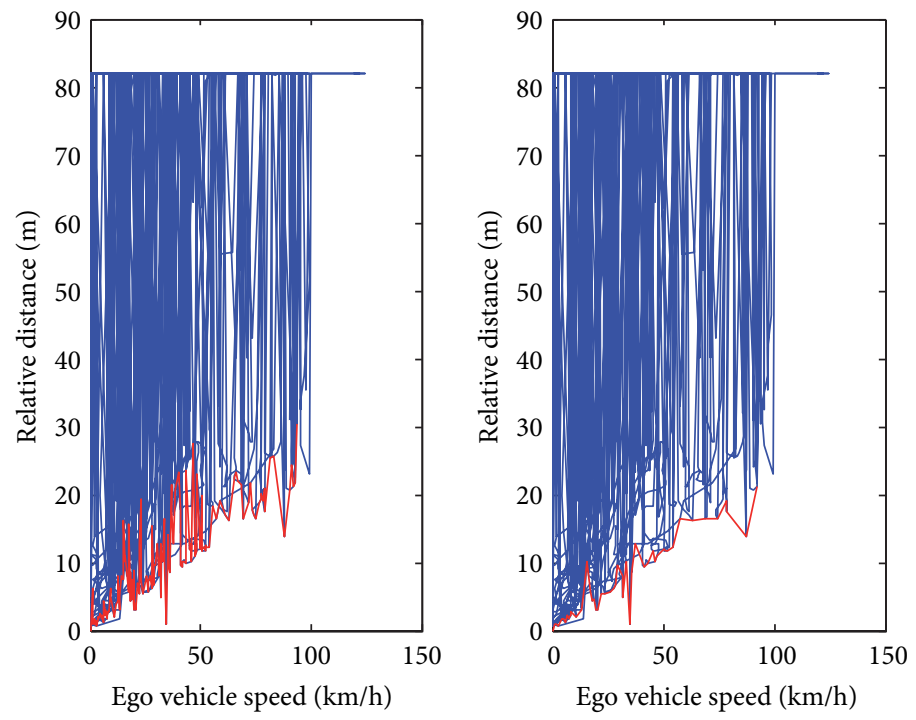

FIgURE 8: Local minimum of relative distance versus ego vehicle speed.

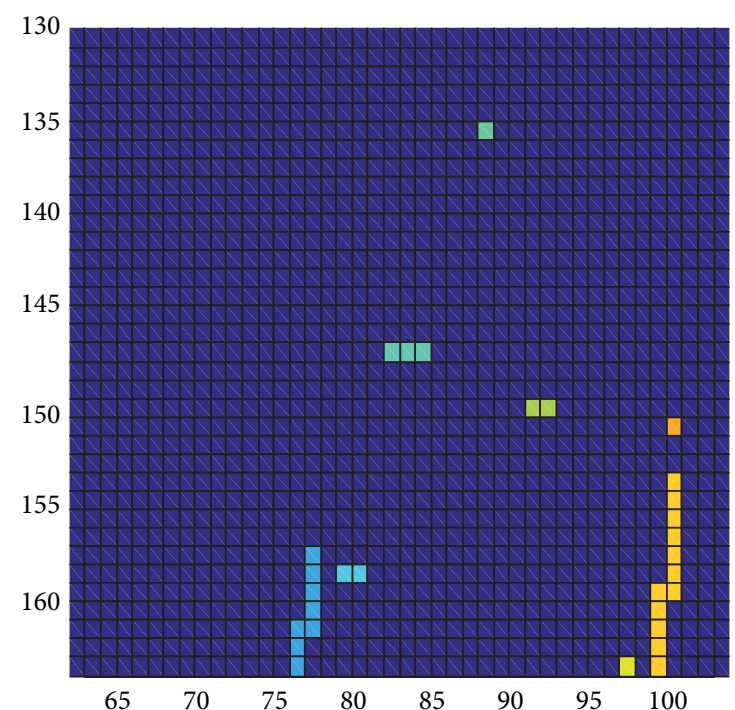

FIGURE 9: Grid-based representation of ego vehicle front.

is greater than a threshold value $D_{\text {thd }}$; that is, if $D\left(r_{i}, r_{i+1}\right)>$ $D_{\text {thd }}$, then the segments are separated. If this is not the case, the segments are not separated.

The following threshold definition taken from [13]:

$$
D_{\text {thd }}=C_{0}+C_{1} \min \left\{r_{i}, r_{i+1}\right\} \text {, }
$$

where $C_{0}$ is a constant parameter used for noise reduction and $C_{1}$ is given by

$$
C_{1}=\sqrt{2(1-\cos \Delta \alpha)}
$$

is used in this paper. The angle $\beta$ in Figure 10 is used to represent the orientation of the detected object [14].

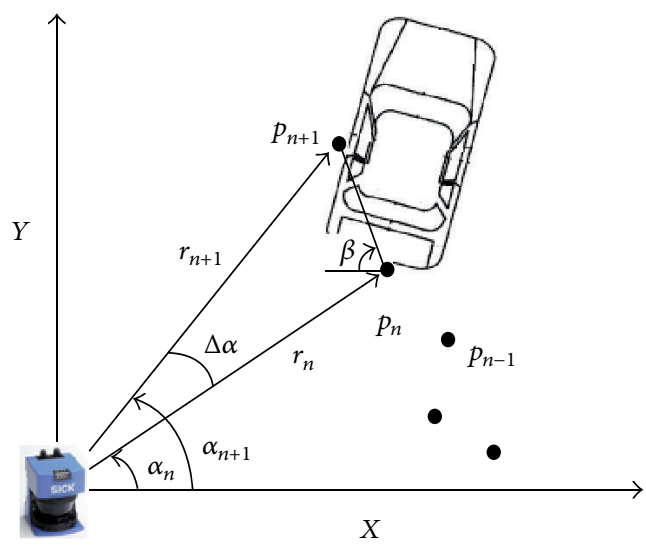

FIGURE 10: Schematical representation of laser scanning and some parameters.

3.3. Detecting Road Limits. Detecting road limits is also important to stay on the road. If it is a barrier or a road limit, the number of scanned points in the vicinity of one $x$-axis position increases as is seen on the left and the right hand sides in Figure 11. The ego vehicle's Lidar is at the point $(x=0, y=0)$ in Figure 11. Both the $x$ and $y$ axes unit is meters. At $x$ axis positions of approximately $-6 \mathrm{~m}$ and $16 \mathrm{~m}$, road limits are observed in Figure 11 in the form of vertical lines.

Road limits can also be recognized by histogram plotting as shown in Figure 12 (see [15] for histogram plotting). At $x$ axis positions that are approximately equal to $-6 \mathrm{~m}$ and $16 \mathrm{~m}$, frequency of reflected laser scanner measurements increases. These positions correspond to the left and right hand road limits.

\section{Kalman Filter Tracking}

The Kalman filter is an algorithm that estimates the state of a dynamic system. The prediction-correction type Kalman 


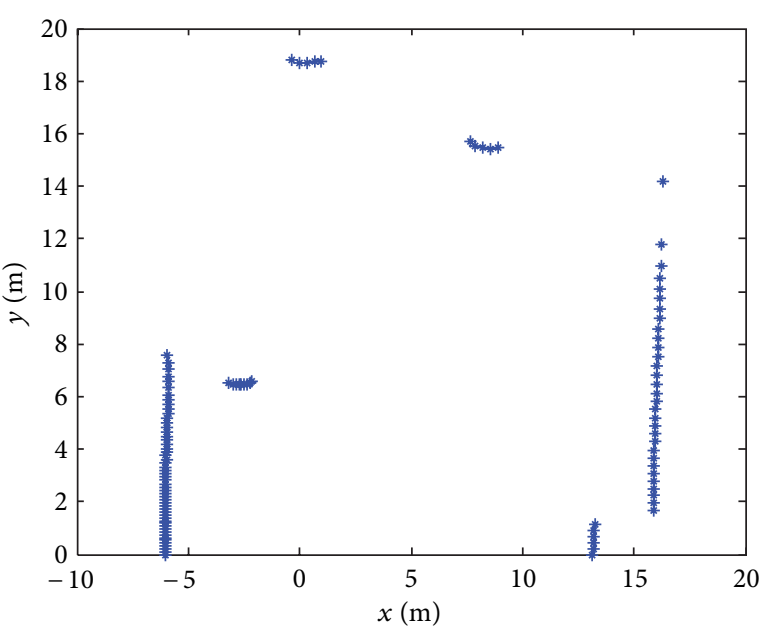

FIgURE 11: Laser scan of ego vehicle.

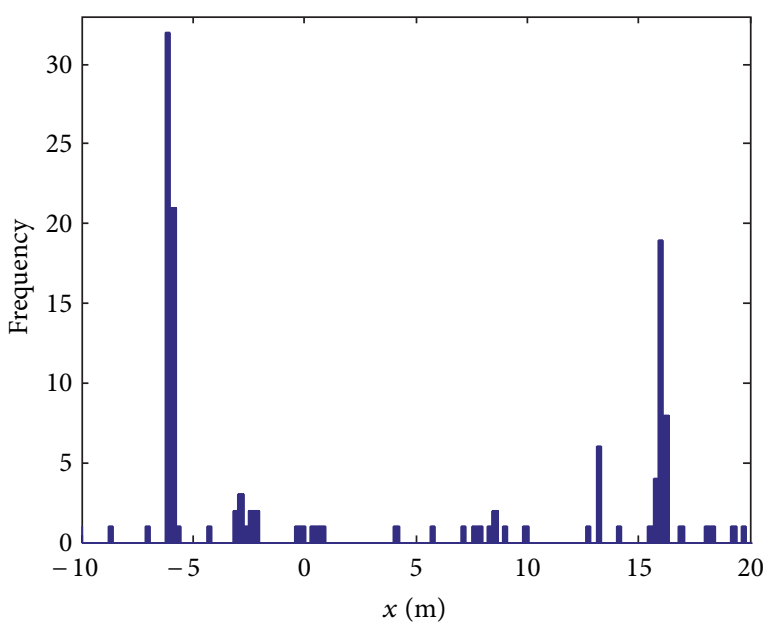

FIGURE 12: Frequency of $x$ coordinates of objects' appearance.

filter algorithm is used to estimate the relative positions, velocities, and uncertainties of moving vehicles in this paper. The prediction part of the Kalman filter produces a search region for each detected vehicle that is then concentrated on in the next laser scan to detect the vehicle with less computational effort. The region that covers the detected vehicle is called the "bounding box." One control point, the average of vehicle scan points, for each bounding box, is considered in the analysis.

4.1. Vehicle Dynamic State. Detected vehicles are assumed to have linear and uniform motion in between two consecutive scans. The dynamics of the vehicles are represented with a constant velocity model as

$$
\begin{aligned}
& x_{k+1}=x_{k}+v_{x k} T_{k}, \\
& y_{k+1}=y_{k}+v_{y k} T_{k} .
\end{aligned}
$$

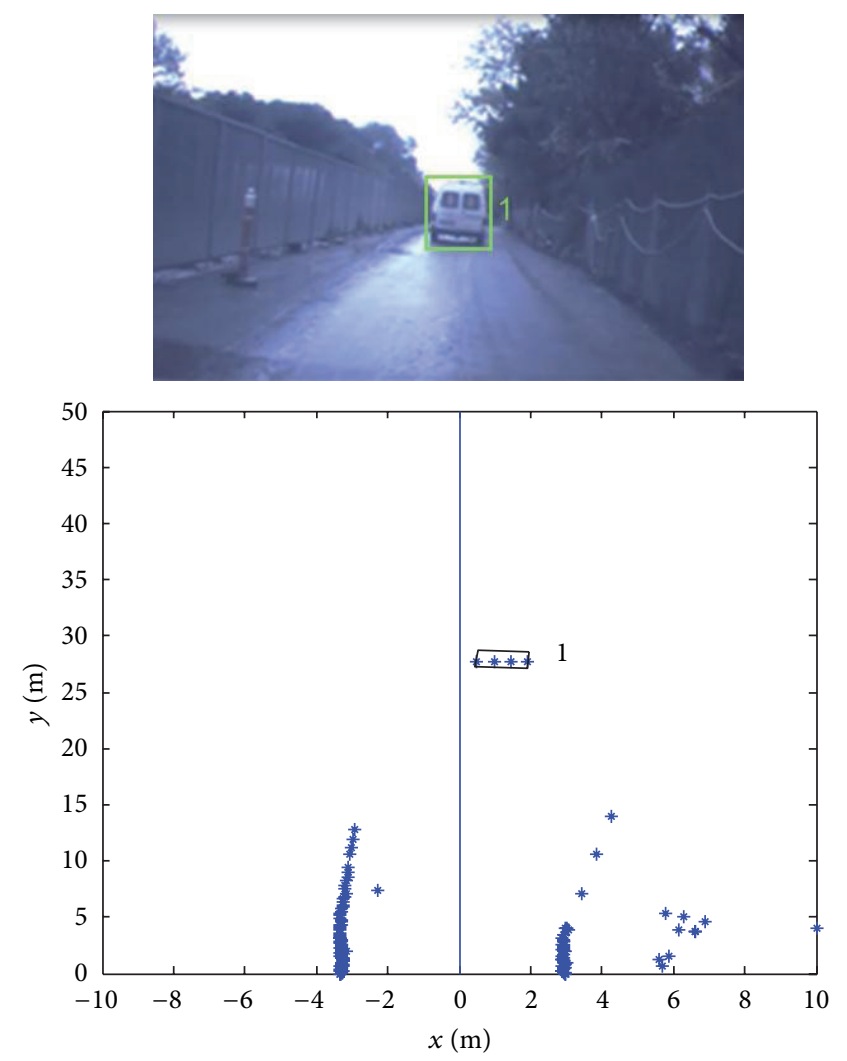

FIGURE 13: Tracking one vehicle.

The subscript $k$ is the current scan number. $x$ and $y$ represent the $x$ and $y$ coordinates of the vehicles. $v_{x}$ and $v_{y}$ are the vehicle speed components along the $x$ and $y$ directions, and $T$ is the time step used. The state space representation of the constant velocity model in (4) is given by (see [14])

$$
X_{k}=\left[\begin{array}{c}
x_{k} \\
y_{k} \\
v_{x k} \\
v_{y k}
\end{array}\right], \quad A_{k}=\left[\begin{array}{cccc}
1 & 0 & T_{k} & 0 \\
0 & 1 & 0 & T_{k} \\
0 & 0 & 1 & 0 \\
0 & 0 & 0 & 1
\end{array}\right]
$$

and the measurement matrix is given by

$$
H=\left[\begin{array}{llll}
1 & 0 & 0 & 0 \\
0 & 1 & 0 & 0
\end{array}\right]
$$

The first two equation, in the state space formulation are (4) while the last two equations represent constant velocity.

4.1.1. Initial Estimates. After the recognition of the vehicles, the current state vector and the covariance matrices are initialized. $Q$ represents the process noise covariance matrix, $R$ represents the measurement noise covariance matrix, and $P$ represents the error covariance. Tracking starts in the next scan after detection. 

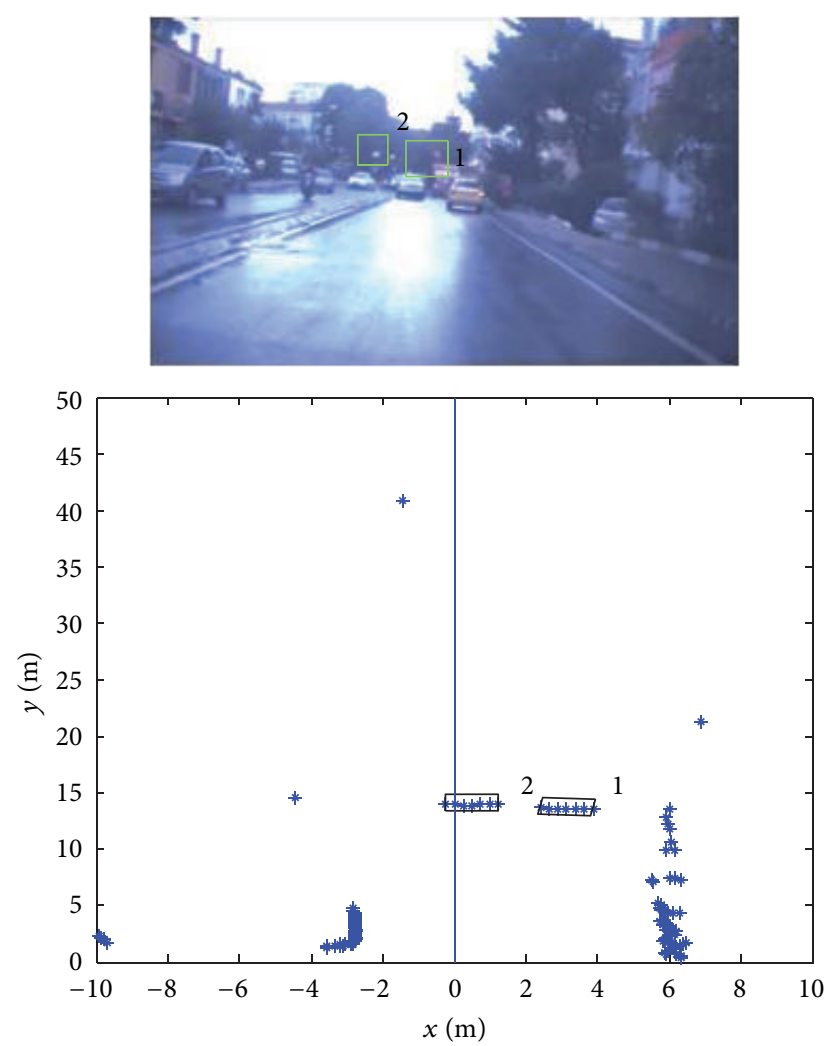

Figure 14: Tracking two vehicles.

4.1.2. Prediction. Prediction is achieved by repeating the prediction equations given below for each frame in the scan sequence:

$$
\begin{gathered}
\widehat{x}_{k}^{-}=A_{k, k-1} \widehat{x}_{k}, \\
P_{k}^{-}=A_{k, k-1} P_{k-1} A_{k, k-1}^{T}+Q_{k} .
\end{gathered}
$$

The position of the detected vehicle is predicted using the model described in (7). Then, the search area for the predicted vehicle position is calculated as mentioned in the hypothesis verification.

4.1.3. Measurement Update (Correction). In the determined search area, the corresponding vehicle is searched by extracting horizontal and vertical edges. The position of the tracked vehicle is updated based on the measurements:

$$
\begin{gathered}
K_{k}=P_{k}^{-} H^{T}\left(H P_{k}^{-} H^{T}+R\right)^{-1}, \\
\widehat{x}_{k}=\widehat{x}_{k}^{-}+K\left(y_{k}-H \widehat{x}_{k}^{-}\right), \\
P_{k}=\left(I-K_{k} H\right) P_{k}^{-1} .
\end{gathered}
$$

In the above equations, $K$ is the filter gain, $y$ is the measurement vector, and $H$ is the measurement matrix (see [16] for more details of the Kalman filter).

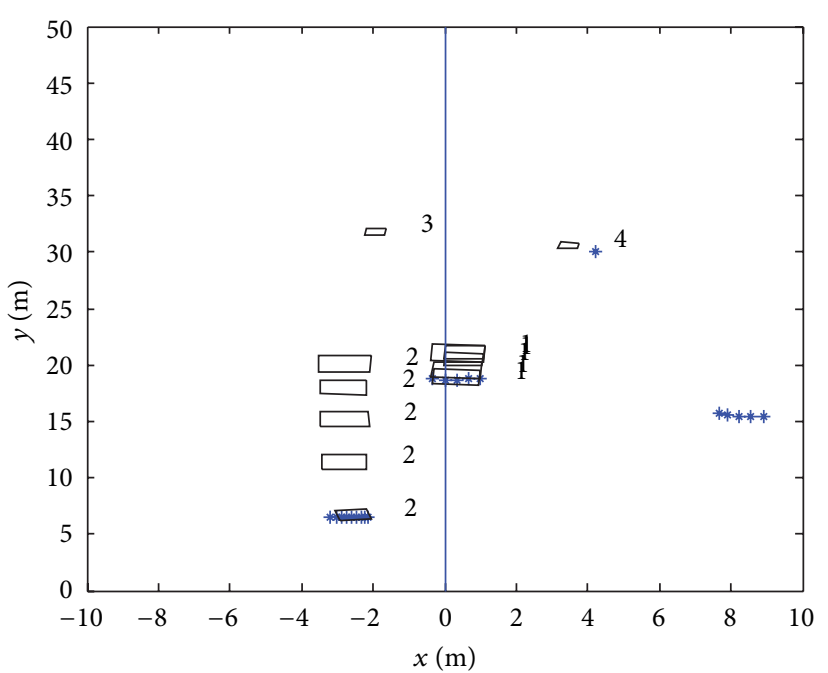

FIgURE 15: Kalman filter tracking of vehicles for $4 \mathrm{sec}$.

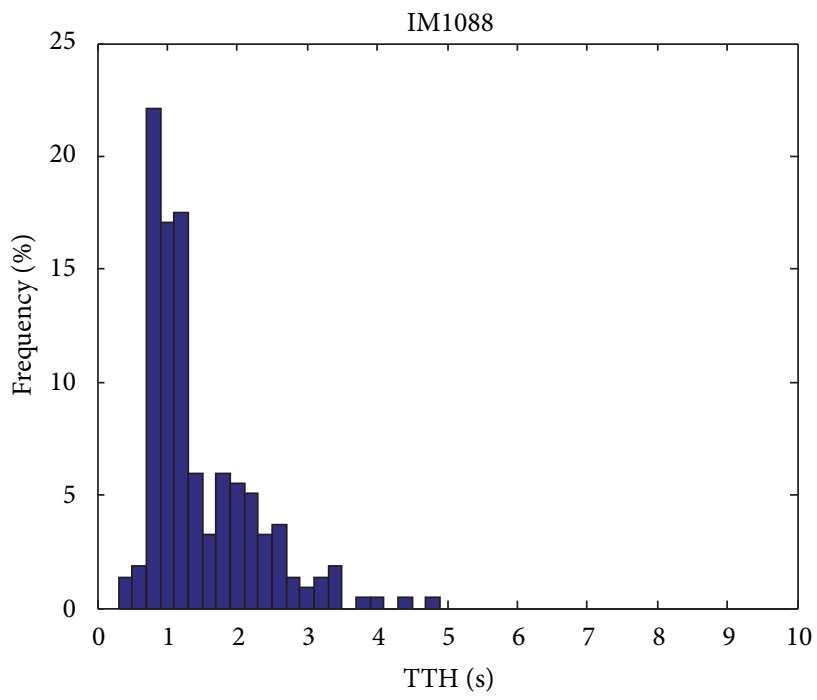

FIGURE 16: TTH histogram for driver IM1088.

\section{Kalman Filter Tracking Results}

Results of Kalman filter tracking of objects in the Lidar data are presented in this section. Corresponding camera images are also presented for better visualisation of the tracked objects. A range of $(-4,+4)$ meters along the $x$ axis and $[0,50]$ meters along the $y$ axis are used for investigation of the Lidar data.

In Figure 13, one object is tracked. Security barriers on the left side of ego vehicle can be seen on the Lidar image. Also a traffic warning pylon is at a point that is approximately at $-2 \mathrm{~m}$ in the $x$ coordinates. The corresponding camera image is shown in the upper subplot of Figure 13. The black box surrounding the vehicle marked with 1 is called the bounding box in the Lidar data [17]. The distance to the tracked vehicle in front is also computed in the Kalman filter algorithm. This 

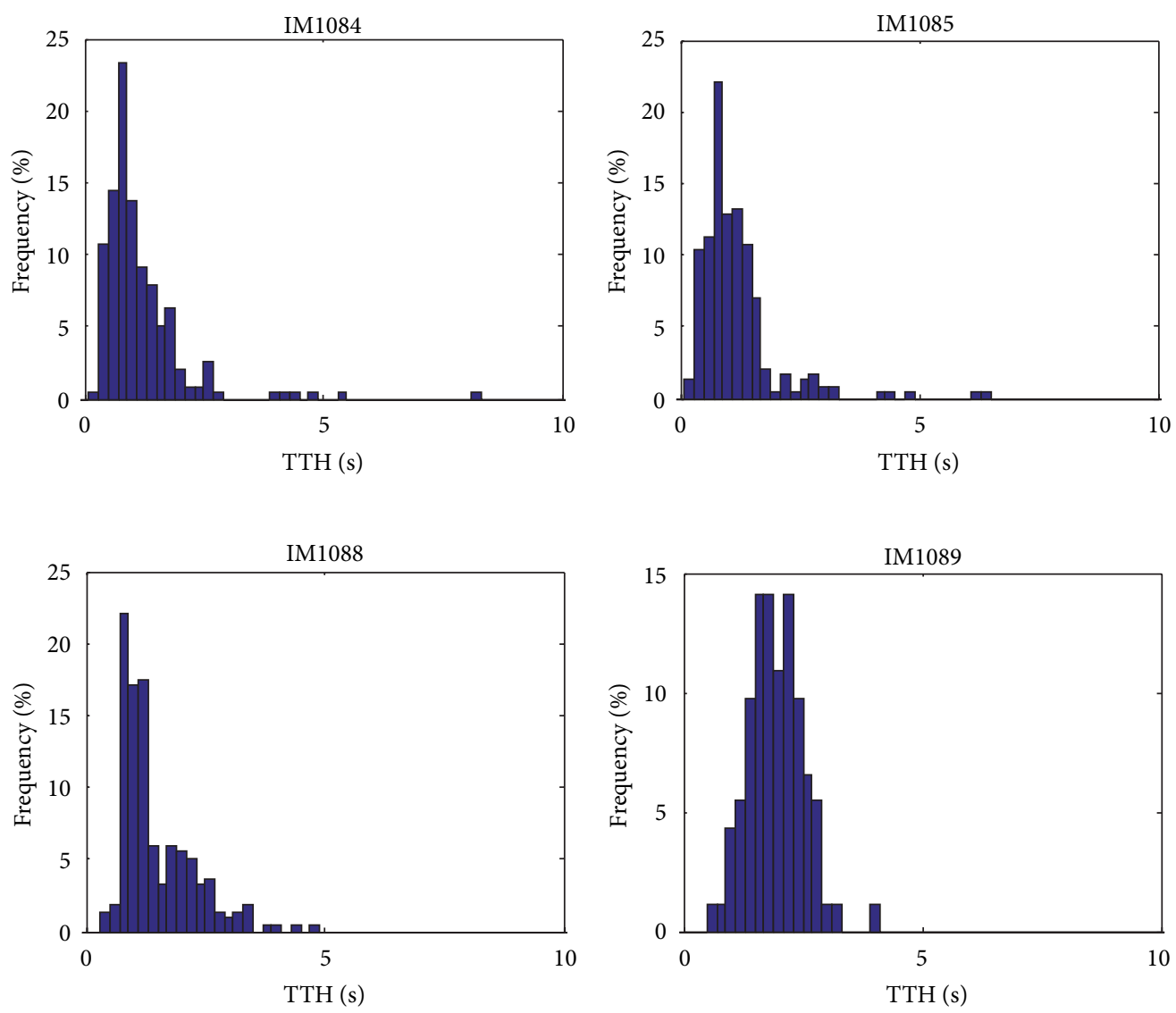

FIGURE 17: TTH histogram for four selected drivers.

distance along with the ego vehicle speed obtained from the vehicle CAN bus is used to compute TTH.

In Figure 14, two vehicles are tracked. The Kalman filter helps to predict where a vehicle possibly exists in the next scan. By defining a search area, the vehicle can be found in the next scan. Figure 15 shows the tracking of the vehicles for $4 \mathrm{sec} .4 \mathrm{scan}$ results are superimposed on each other for $4 \mathrm{sec}-$ onds at an update rate of $1 \mathrm{~Hz}$. Blue dots are used to illustrate the initial state of the Lidar scan. The first and second vehicles are faster than the ego vehicle. The third and fourth vehicles entered the scanning range of the Lidar at the fourth second. During these computations, the nearest vehicle in front is the vehicle being detected and tracked. Its distance to the ego vehicle is used in the subsequent TTH computations.

\section{Results and Discussion}

Figure 16 shows the TTH histogram for driver IM1088 where the Kalman filter-based algorithm was used to detect the nearest vehicle in front and the distance to it. The TTH histogram in Figure 16 has a peak at less than $1 \mathrm{sec}$. of TTH showing that this driver has a tendency of driving too close to the vehicle in front.

Figure 17 shows the TTH histograms of four selected drivers in the data set of the DriveSafe project. TTH statistical information of the selected four drivers is presented in Table 1. Driver IM1089 drives more safely than the others as he uses a larger TTH value with a mean of $2.031 \mathrm{sec}$. Driver IM1084 drives more dangerously as compared to the others with a mean TTH of $1.247 \mathrm{sec}$.

\section{Conclusions}

In this paper, the Lidar data collected during the DriveSafe project was used to investigate driver behavior and to analyze the vehicle's surrounding environment. Time to headway was investigated to obtain information about the driver. Lidar data is used to obtain information on what lies ahead of the ego vehicle. Objects in front of the ego vehicle are detected with grid-based and point distance-based segmentation methods. Then, the point distance-based method is selected for tracking of detected vehicles. Tracking of these objects is carried out with the Kalman filter using the constant speed model. The relative position and velocities of these tracked vehicles were estimated using the Kalman filter. The method was applied to example driving data in the DriveSafe project database.

The method used in this paper for TTH computation will be used in our future studies to investigate all the driver data in the DriveSafe project database. 


\section{Acknowledgments}

The data used in the analyses presented in this paper was from one of the drivers who took part in the DriveSafe project data collection experiments. The authors would, thus, like to thank the Turkish National Planning Association (DPT) for funding the DriveSafe project (EACF05-00322/1) and all the project partners of the DriveSafe project.

\section{References}

[1] National Center for Statistics and Analysis, National Highway Traffic Safety Administration, http://www.nhtsa.gov/.

[2] International Traffic Safety Data and Analysis Group, http:// internationaltransportforum.org/.

[3] T. Wakita, K. Ozawa, C. Miyajima et al., "Driver identification using driving behavior signals," IEICE Transactions on Information and Systems, vol. E89-D, no. 3, pp. 1188-1194, 2006.

[4] J. Archer and I. Kosonen, "The potential of micro-simulation in relation to traffic safety assesment," ESS Conference Proceedings, vol. 45, no. 7, pp. 569-590, 2006.

[5] O. Gietelink, J. Ploeg, B. de Schutter, and M. Verhaegen, "Development of advanced driver assistance systems with vehicle hardware-in-the-loop simulations," Vehicle System Dynamics, vol. 44, no. 7, pp. 569-590, 2006.

[6] B. Aytekin, E. Dinçmen, B. A. Güvenç et al., "Framework for development of driver adaptive warning and assistance systems that will be triggered by a driver inattention monitor," International Journal of Vehicle Design, vol. 52, no. 1-4, pp. 20-37, 2010.

[7] H. Abut, H. Erdoğan, A. Erçil et al., "Data collection with uyanik: too much pain, but gains are coming," in Biennial on DSP For in-Vehicle and Mobile Systems, 2007.

[8] I. M. C. Uygan, K. Kahraman, R. Karaahmetoglu et al., "Cooperative adaptive cruise control implementation of team mekar at the grand cooperative driving challenge," Special Issue of the IEEE Transactions on Intelligent Transportation Systems on Implementations of Cooperative Adaptive Cruise Control at the Grand Cooperative Driving Challenge, vol. 13, no. 3, pp. 10621074, 2012.

[9] L. Neubert, L. Santen, A. Schadschneider, and M. Schreckenberg, "Single-vehicle data of highway traffic: a statistical analysis," Physical Review E, vol. 60, no. 6, pp. 6480-6490, 1999.

[10] R. Riccardo and G. Massimiliano, "An empirical analysis of vehicle time headways on rural two-lane two-way roads," in Proceedings of the 15th meeting of the EURO Working Group on Transportation (EWGT '12), September 2012.

[11] N. Tricot, B. Rajaonah, M. P. Pacaux, and J. C. Popieul, "Driver's behaviors and human-machine interactions characterization for the design of an advanced driving assistance system," in Proceedings of the IEEE International Conference on Systems, Man and Cybernetics (SMC '04), pp. 3976-3981, Valenciennes, France, October 2004.

[12] P. Lindner and G. Wanielik, "3D LIDAR processing for vehicle safety and environment recognition," in Proceedings of the IEEE Workshop on Computational Intelligence in Vehicles and Vehicular Systems (CIVVS '09), pp. 66-71, Nashville, Tenn, USA, April 2009.

[13] K. C. J. Dietmayer, J. Sparbert, and D. Streller, "Model based object classification and object tracking in traffic scenes from range images," in Proceedings of the 4th IEEE Intelligent Vehicles Symposium, Tokyo, Japan, 2001.
[14] C. Premebida and U. Nunes, "Segmentation and geometric extraction from 2D laser range data for mobile robot applications," in Proceedings of the 4th National Festival of Robotics Scientific Meeting (ROBOTICA '05), pp. 17-25, Coimbra, Portugal, 2005.

[15] F. Fayad and V. Cherfaoui, "Tracking objects using a laser scanner in driving situation based on modeling target shape," in Proceedings of the IEEE Intelligent Vehicles Symposium (IVS '07), pp. 44-49, Istanbul, Turkey, June 2007.

[16] B. Aytekin and E. Altuğ, "Increasing driving safety with a multiple vehicle detection and tracking system using ongoing vehicle shadow information," in Proceedings of the IEEE International Conference on Systems, Man and Cybernetics (SMC '10), pp. 3650-3656, Istanbul, Turkey, October 2010.

[17] D. Streller, K. Fürstenberg, and K. Dietmayer, "Vehicle and object models for robust tracking in traffic scenes using laser range images," in Proceedings of the IEEE 5th International Conference on Intelligent Transportation Systems, pp. 118-123, 2002. 

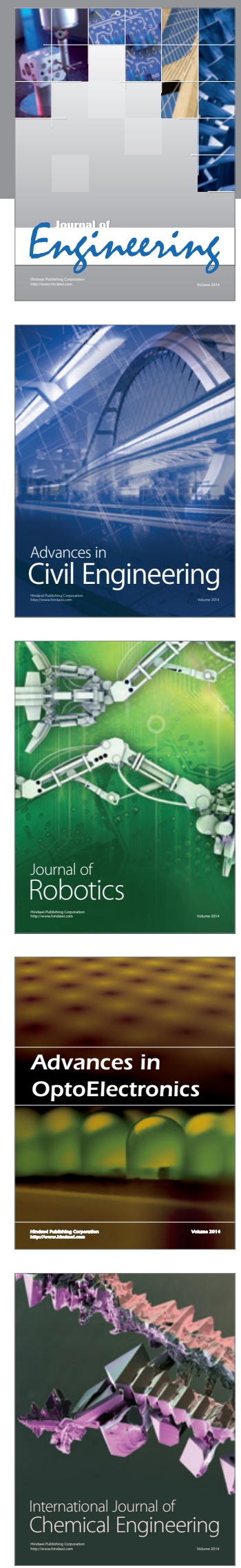

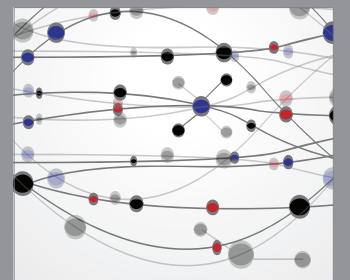

The Scientific World Journal
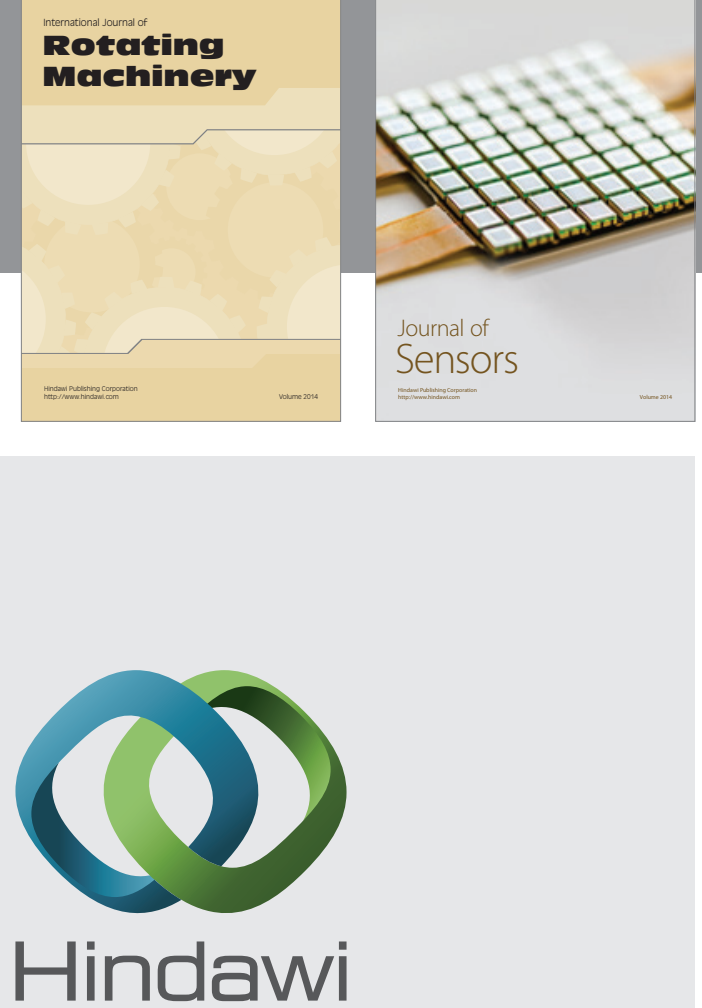

Submit your manuscripts at http://www.hindawi.com
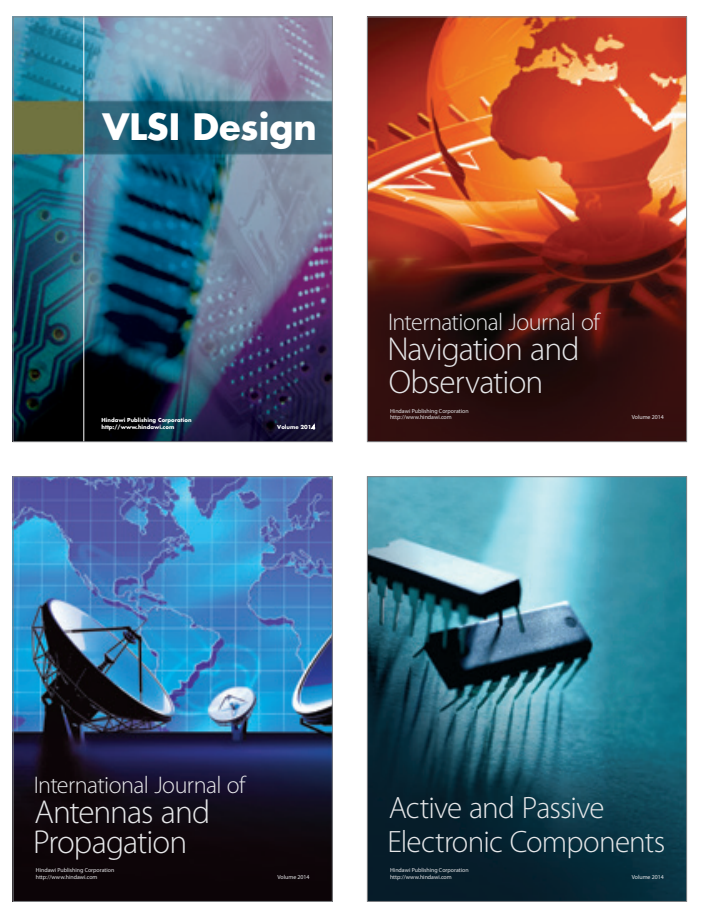
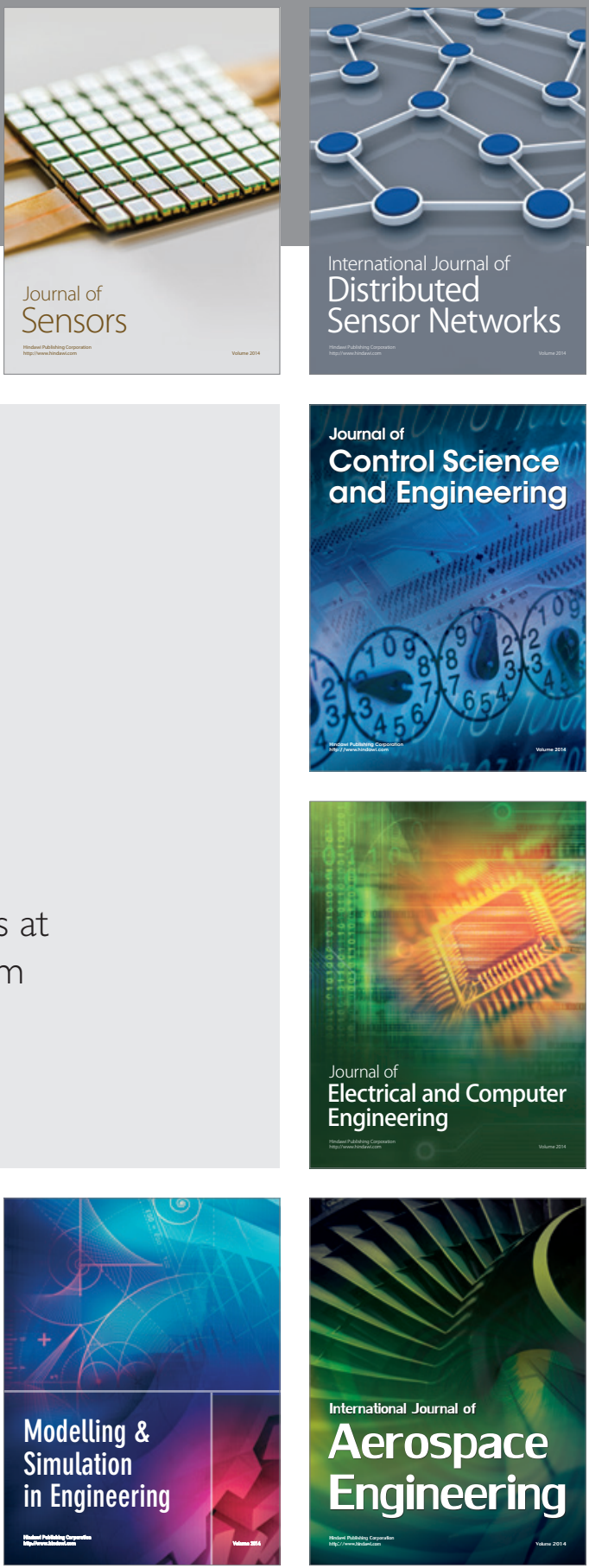

Journal of

Control Science

and Engineering
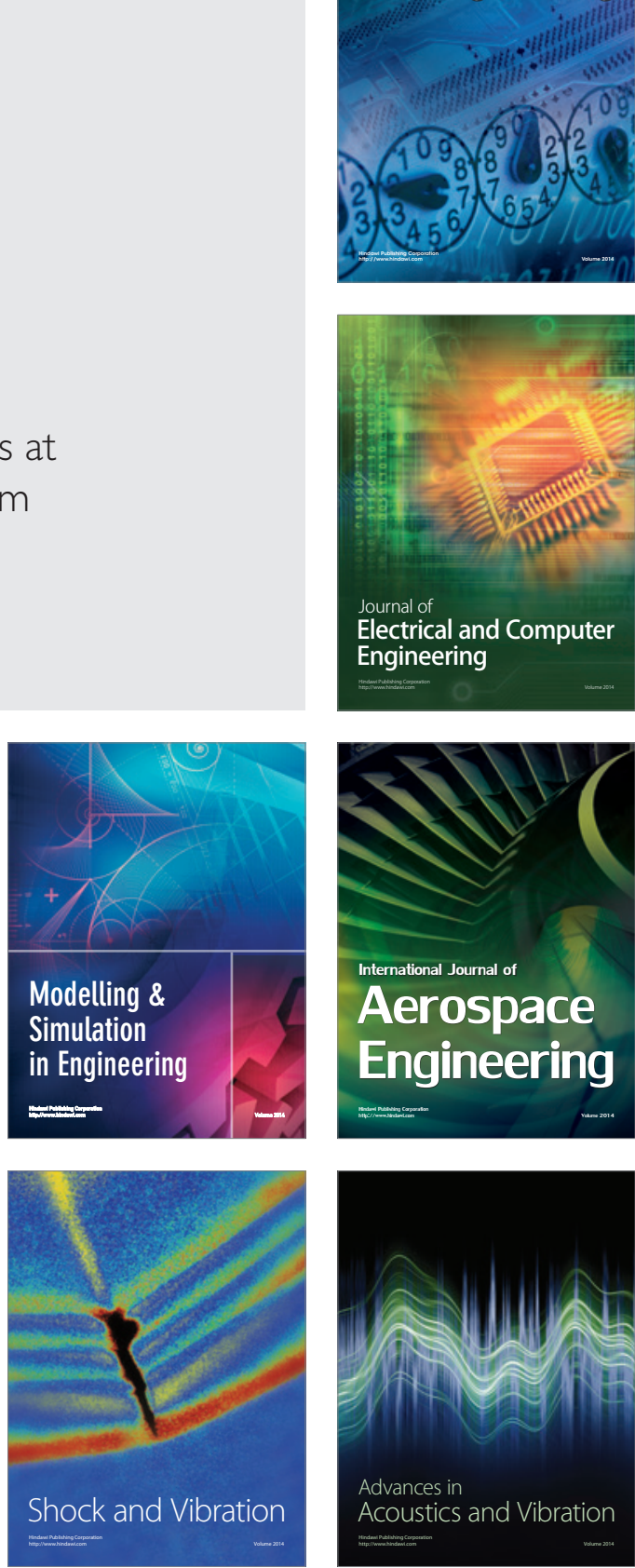